\title{
Laënnec HURBON
}

docteur en Théologie (Institut catholique de Paris) et en Sociologie (Sorbonne), directeur de recherche au CNRS et professeur à I'Université Quisqueya de Port-au-Prince

\section{(1999)}

\section{"Jean Benoist. Hindouismes créoles. Mascareignes, Antilles."} COMPTE RENDU DE LIVRE

Un document produit en version numérique par Jean-Marie Tremblay, bénévole, professeur de sociologie au Cégep de Chicoutimi

Courriel: jean-marie_tremblay@uqac.ca

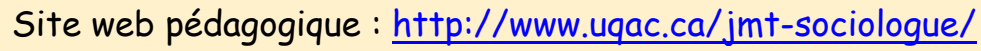

Dans le cadre de: "Les classiques des sciences sociales" Une bibliothèque numérique fondée et dirigée par Jean-Marie Tremblay, professeur de sociologie au Cégep de Chicoutimi

Site web: http://classiques.uqac.ca/

Une collection développée en collaboration avec la Bibliothèque

Paul-Émile-Boulet de l'Université du Québec à Chicoutimi Site web: http://bibliotheque.ugac.ca/ 


\section{Politique d'utilisation de la bibliothèque des Classiques}

Toute reproduction et rediffusion de nos fichiers est interdite, même avec la mention de leur provenance, sans l'autorisation formelle, écrite, du fondateur des Classiques des sciences sociales, Jean-Marie Tremblay, sociologue.

Les fichiers des Classiques des sciences sociales ne peuvent sans autorisation formelle:

- être hébergés (en fichier ou page web, en totalité ou en partie) sur un serveur autre que celui des Classiques.

- servir de base de travail à un autre fichier modifié ensuite par tout autre moyen (couleur, police, mise en page, extraits, support, etc...),

Les fichiers (.html, .doc, .pdf, .rtf, .jpg, .gif) disponibles sur le site Les Classiques des sciences sociales sont la propriété des Classiques des sciences sociales, un organisme à but non lucratif composé exclusivement de bénévoles.

Ils sont disponibles pour une utilisation intellectuelle et personnelle et, en aucun cas, commerciale. Toute utilisation à des fins commerciales des fichiers sur ce site est strictement interdite et toute rediffusion est également strictement interdite.

L'accès à notre travail est libre et gratuit à tous les utilisateurs. C'est notre mission.

Jean-Marie Tremblay, sociologue

Fondateur et Président-directeur général, LES CLASSIQUES DES SCIENCES SOCIALES. 
Cette édition électronique a été réalisée par Jean-Marie Tremblay, bénévole, professeur de sociologie au Cégep de Chicoutimi à partir de :

Laënnec HURBON

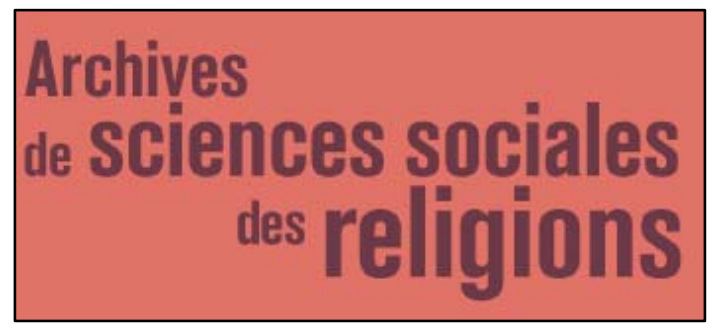

"Jean Benoist. Hindouismes créoles, Mascareignes, Antilles. Paris : Éditions du Comité des Travaux historiques et scientifiques, 1998, 303p. (illustr.., index)."

Un texte publié dans la revue Archives des sciences sociales des religions, vol. 108, no 1, 1999, pp. 47-48. Compte rendu de livre.

http://www.persee.fr/web/revues/home/prescript/article/assr_03355985_1999_num_108_1_1097_t1_0047_0000_2

[Autorisation formelle accordée par l'auteur le 19 mai 2009 de diffuser toutes ses publications dans Les Classiques des sciences sociales.]

Courriel : Ihurbon@yahoo.com

Polices de caractères utilisée : Comic Sans, 12 points.

Édition électronique réalisée avec le traitement de textes Microsoft Word 2008 pour Macintosh.

Mise en page sur papier format : LETTRE US, $8.5^{\prime \prime} \times 11^{\prime \prime}$.

Édition numérique réalisée le 28 avril 2011 à Chicoutimi, Ville de Saguenay, Québec.

Fait avec

Macintosh 


\section{Laënnec HURBON}

docteur en Théologie (Institut catholique de Paris) et en Sociologie (Sorbonne), directeur de recherche au CNRS et professeur à l'Université Quisqueya de Port-au-Prince Doyen

"Jean Benoist. Hindouismes créoles, Mascareignes, Antilles. Paris: Éditions du Comité des Travaux historiques et scientifiques, 1998, 303p. (illustr., index)."

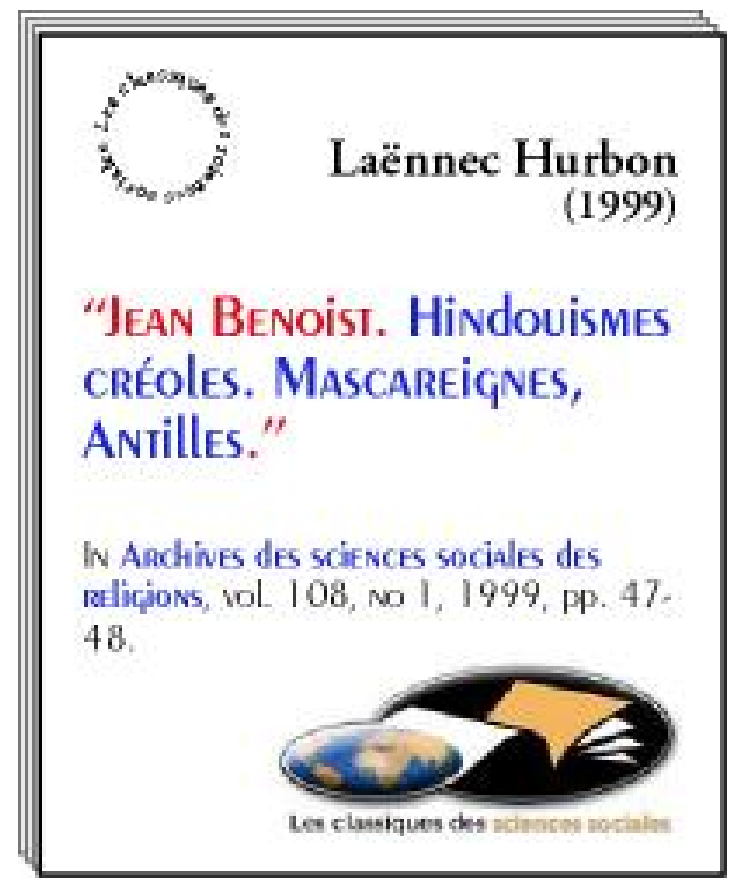

Un texte publié dans la revue Archives des sciences sociales des religions, vol. 108, no 1, 1999, pp. 47-48. Compte rendu de livre. 


\section{Laënnec HURBON}

"Jean Benoist. Hindouismes créoles, Mascareignes, Antilles.

Paris : Éditions du Comité des Travaux historiques et scientifiques, 1998, 303p. (illustr.. index)."

Un texte publié dans la revue Archives des sciences sociales des religions, vol. 108, no 1, 1999, pp. 47-48. Compte rendu de livre.

Vers le milieu du XIXe siècle, alors que l'abolition de l'esclavage est à l'ordre du jour, les empires français et britanniques organisent une nouvelle migration à partir de l'Inde vers les îles de l'Océan indien (Réunion, Maurice) et les Antilles (ou la Caraïbe, comme la Guadeloupe et la Martinique). Les anciens esclaves noirs désertant les plantations et les habitations sucrières, il fallait trouver une main d'oeuvre plus disponible: ce sont les Indiens du Sud de l'Inde qui recevront le statut « d'engagés » et devront assurer la continuation de la société de plantation mais sous de nouvelles formes plus acceptables et moins scandaleuses. Les Indiens allaient ainsi s'installer dans un milieu porté à les soupçonner de connivence avec le pouvoir, pendant que celui?ci les privait de leurs droits civiques et les maintenait au plus bas de l'échelle sociale. Pour construire et sauvegarder une identité, ils ont dû recourir à I'hindouisme qu'ils acclimatent et adaptent dans le contexte colonial des îles. Leur mode de vie, leur système de valeurs et de représentations étaient peu étudiés jusque vers les années 1970. Le nouvel ouvrage que J.B. vient de publier sur les croyances et pratiques religieuses héritées de l'Inde et qu'il appelle avec justesse "Hindouismes créoles » est d'un apport considérable à la connaissance des sociétés créoles dont l'histoire, croyait-on, apparaissait jusqu'ici dominée par l'opposition maîtres/ esclaves et blancs/ noirs. 
La longue fréquentation du terrain dont témoigne l'auteur et surtout son autorité dans le champ des sciences sociales de la Caraïbe et de l'Océan indien avec les travaux pionniers que sont par exemple L'archipel inachevé et Anthropologie médicale en société créole auguraient du succès de sa recherche sur la part indienne des cultures créoles. J.B. commence par nous introduire dans la problématique générale des migrations indiennes dans le monde pour mieux faire saisir la spécificité de la condition des «engagés » dans l'Océan indien et dans les Antilles. L'Inde qu'il essaie d'étudier est «I'Inde dominée » (p. 20) qui va cependant réussir à s'affirmer dans le contexte rigoureusement hostile du système de plantation. Quelle continuité peut-on découvrir entre le panthéon de l'Inde et celui des migrants dans les sociétés d'accueil ? Quelles sont les sources réelles de leurs croyances et pratiques? Plus précisément, quelle place ont les cultes populaires de l'Inde et les grands textes sacrés de la tradition sanscrite dans les hindouismes créoles? L'investigation est menée de telle manière qu'en aucun cas I' $A$. ne détache le rituel et les croyances de leurs sources et en même temps des conditions spéciales de leur évolution dans la société globale. Le sacré est constamment décrit et analysé dans son articulation au social. Justement chaque élément de la vie religieuse indienne renvoie à un élément de la vie sociale qu'il fonde et consolide : la hiérarchisation des dieux correspond à celle des groupes sociaux (famille, castes et tribus) : "I'arborescence du divin, écrit $J . B .$, fonctionne comme une échelle symbolique du social à laquelle on monte d'étage en étage » (p. 48). Sur ce principe structural, l'A. parvient à montrer comment le mode d'organisation des temples (en grands temples urbains dédiés par les commerçants et non par les « engagés » aux cultes majeurs comme au Dieu Siva, puis en temples des plantations ou chapelles indiennes établies sur les plantations pour les engagés, et enfin en temples personnels destinés aux pratiques familiales) renvoie à celui du panthéon. Dans le contexte des sociétés créoles, [48] apparaissent beaucoup plus importants les cultes rendus dans les temples de plantations. Ainsi par exemple Mariamin (ou Ma- 
liémé) est le nom de la divinité la plus populaire aussi bien à la Réunion et Maurice qu'en Guadeloupe et Martinique. Elle a une double face, négative comme agent de mal ou de maladies et positive comme agent de guérison et de protection. C'est à ce niveau qu'on peut comprendre comment la religion est liée indissolublement aux représentations de la maladie et aux pratiques thérapeutiques. Parmi les sources des maladies, il y a des « démons » et des « mauvais esprits» qui sont parfois tout simplement des âmes errantes de mauvais morts et qu'il faut savoir exorciser. On peut bien comprendre que dans la situation d'oppression de la société créole prospèrent ces « mauvais esprits » et que le migrant indien est ainsi constamment porté à s'attacher à la religion indienne.

Il n'est pas possible ici de rendre compte de toute la richesse de cet ouvrage qui nous offre une description rigoureuse des grandes cérémonies populaires, de leur calendrier, de leur impact sur la vie quotidienne de la diaspora indienne des îles de l'Océan indien et de la Caraïbe. En particulier, nous devons signaler l'étude anthropologique qui est faite des deux grandes épreuves physiques qui structurent les cérémonies, à savoir la marche dans le feu et les piqûres sur la langue et sur le thorax qui, bien assumées, expriment l'attachement de l'individu à la divinité et sa reconnaissance envers elle pour les protections qu'elle a su procurer. Qu'il nous suffise d'attirer l'attention sur les questions théoriques soulevées dans ce travail et qui ont la vertu de féconder la recherche en anthropologie et en sociologie des sociétés créoles. Au départ, J.B. ouvre une réflexion qui est capitale dans l'évolution actuelle du monde contemporain sur les rapports entre ethnicité et culture. En effet, ce qui apparaît dans l'étude des modes de résistance des migrants indiens à l'oppression et au mépris à caractère raciste c'est que la religion indienne a pu s'adapter et servir de base à la reconstruction d'une identité, pendant qu'elle apparaissait peu à peu déliée de l'indianité. On dirait que le problème d'une humanité libérée du carcan des représentations ethnicistes et «racistes » de l'identité émergeait déjà fort tôt dans toute son acuité dans les 
sociétés créoles. D'un autre côté, I'A. nous invite à revisiter et à repenser le syncrétisme. Jusqu'ici l'anthropologie connaissait les pratiques d'annexion des symboles du catholicisme par les religions afroaméricaines (comme le vaudou haïtien et le candomblé brésilien). La diaspora indienne dans les sociétés créoles n'a pas eu de peine à accueillir le Dieu et les saints du catholicisme comme des éléments tout à fait intégrables dans la hiérarchie des divinités indiennes, sans que cela suppose la dissolution de la religion indienne. Le clergé catholique avait beau combattre I'hindouisme comme un culte diabolique, il ne parvenait guère à convaincre le migrant indien à l'abandon de ses croyances. Bien plus, il se produit aujourd'hui un effort de ressourcement en Inde même. Et l'on s'aperçoit que les préjugés nourris jusqu'ici contre les cultes indiens des sociétés créoles disparaissent au fur et à mesure qu'on découvre la variété déjà en Inde même des cultes qui ne se réduisent pas à ce que prescrivent les textes sacrés de la tradition sanscrite. En revanche, J.B. ne s'est pas arrêté à une vision fixiste des hindouismes créoles, il prend le risque d'interroger les tendances actuelles à faire du culte un étendard de l'affirmation identitaire et de la promotion sociale, la diaspora indienne entrant de plain-pied dans le processus de mondialisation sans complexe. Mais tout se passe en même temps comme si la crise qui affectait le catholicisme en s'ouvrant aux réalités sociales et culturelles finissait par atteindre également les pratiquants des cultes populaires indiens. Aussi sectes et nouveaux mouvements religieux sont-ils les nouveaux lieux d'accueil de tous ceux qui ne parviennent pas à assumer l'évolution actuelle du catholicisme et de I'hindouisme.

Laënnec Hurbon.

In Archives des sciences sociales des religions

Fin du texte 